\title{
ESTIMATIVAS DE PARÂMETROS GENÉTICOS E PREDIÇÃO DE VALORES GENOTÍPICOS NO MELHORAMENTO DO CAFEEIRO PELO PROCEDIMENTO REML/BLUP ${ }^{(1)}$
}

\author{
MARCOS DEON VILELA DE RESENDE ${ }^{(2)} ;$ ENES FURLANI-JÚNIOR $^{(3)}$; \\ MÁRIO LUÍZ TEIXEIRA DE MORAES ${ }^{(3)}$; LUIZ CARLOS FAZUOLI $^{(4)}$ \\ RESUMO
}

\begin{abstract}
Objetivou-se aplicar o método REML/BLUP em programas de melhoramento genético do cafeeiro, utilizando-o na estimação de parâmetros genéticos e predição de valores genotípicos para a espécie Coffea arabica. O experimento foi instalado em julho de 1998 pela Universidade Estadual Paulista (UNESP), em Selvíria (MS). As 12 cultivares selecionadas pelo Instituto Agronômico (IAC), Campinas (SP), foram avaliadas no delineamento em blocos ao acaso com quatro repetições e dez plantas por parcela. Os resultados revelaram baixa variabilidade genética entre as cultivares para os caracteres altura da planta, diâmetro do caule e número de ramos plagiotrópicos, avaliados aos 26 meses. Apenas as cultivares Catuaí Amarelo, Icatu Vermelho e Catuaí Vermelho apresentaram valores genotípicos preditos para o diâmetro do caule superiores à média geral desse caráter. A acurácia na avaliação dos valores genotípicos das cultivares para o caráter diâmetro do caule equivaleu, em média, a 76\%. A adoção de uma experimentação com duas plantas por parcela e 20 repetições poderá elevar a $90 \%$ a acurácia seletiva para o caráter diâmetro do caule. O método de modelos mistos (REML/BLUP) mostrou-se adequado à estimação de parâmetros genéticos e predição de valores genotípicos no melhoramento do cafeeiro, podendo ser empregado rotineiramente.

Palavras-chave: melhoramento de Coffea arabica, modelos lineares mistos, componentes de variância, melhoramento de plantas perenes, predição de variáveis aleatórias, seleção.
\end{abstract}

\section{ABSTRACT \\ ESTIMATION OF GENETIC PARAMETERS AND PREDICTION OF GENOTYPIC VALUES IN COFFEE BREEDING BY THE REML/BLUP METHOD}

The aim of this paper is to apply the REML/BLUP methodology in coffee breeding programs, using it in the parameter estimation and genotypic values prediction in a cultivar trial of Coffea arabica. A trial established in Selvíria-MS by UNESP/Campus de Ilha Solteira, was studied. The trial evaluated 12 cultivars developed by the Instituto Agronômico de Campinas in a complete block design with four replications and 10 plants per plot. The results showed a low genetic variability among cultivars for the traits height, stem diameter and number of primary branches, at age of 26 months. Only the cultivars Catuaí Amarelo, Icatu Vermelho and Catuaí Vermelho showed predicted genotypic values for stem diameter superior to the general mean of the trait. The accuracy of the genotypic evaluation of the cultivars for stem diameter was, in average, $76 \%$. The use of an experimental design with two plants per plot and 20 replications can lead to an accuracy of $90 \%$ for stem diameter. The mixed model

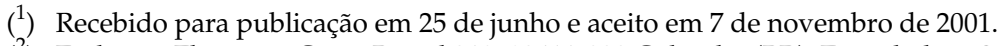

(2) Embrapa Florestas, Caixa Postal 319, 83411-000 Colombo (PR). E-mail: deon@cnpf.embrapa.br

$\left.{ }^{3}\right)$ Departamento de Fitotecnia, Faculdade de Engenharia de Ilha Solteira, Universidade Estadual Paulista (FEIS/UNESP), Campus de Ilha Solteira, Caixa Postal 31, 15385-000 Ilha Solteira (SP).

( $\left.{ }^{4}\right)$ Centro de Café e Plantas Tropicais, Instituto Agronômico (IAC), Caixa Postal 28, 13001-970 Campinas (SP). 
methodology (REML/BLUP) showed to be adequate for genetic parameter estimation and genotypic values prediction in coffee breeding and then, could be used routinely.

Key words: Coffea arabica breeding, mixed linear models, variance components, perennial plants breedin, prediction of random variables, selection.

\section{INTRODUÇÃO}

O café é uma das principais fontes de divisas para o Brasil. As duas espécies de cafeeiro mais cultivadas no mundo são Coffea arabica L. - café arábica - e Coffea canephora Pierre - café robusta -, as quais respondem por cerca de $70 \%$ e $30 \%$ da produção mundial. No Brasil, a principal espécie plantada é C. arabica (sobretudo nos Estados de Minas Gerais, São Paulo e Paraná), sendo C. canephora a que apresenta área de plantio mais restrita (Espírito Santo, Rondônia e Sul da Bahia). Em nível mundial, C. arabica é produzida principalmente no Brasil, Colômbia, Quênia e Índia, e C. canephora, no Vietnã, Indonésia, Brasil e Costa do Marfim (FAZUOLI, 1986).

C. arabica é uma espécie autógama alopoliplóide (tetraplóide) com $2 \mathrm{n}=4 \mathrm{x}=44$ cromossomos e $C$. canephora, alógama diplóide com $2 \mathrm{n}=2 \mathrm{x}=22$ cromossomos. C. arabica apresenta distribuição geográfica natural restrita aos países Etiópia (região Sudeste), Sudão (região Sudeste) e Quênia (região Norte), em altitudes entre 1.000 e 2.000 metros. Por outro lado, $C$. canephora tem ampla distribuição geográfica, ocorrendo em regiões de pequena altitude no oeste e centro do continente africano (CARVALHO et al., 1985).

Em razão da importância da cultura do café para o Brasil, os programas de melhoramento do cafeeiro desenvolvidos no País apresentam importância fundamental como fator de aumento da produção, produtividade e de desenvolvimento sócio-econômico das regiões de cultivo. Tais programas têm sido desenvolvidos com sucesso para C. arabica (FAzUOLI, 1986; Carvalho e Fazuoli, 1993; Sera, 1998; Sakiyama et al., 1999; Mendes, 1999) e C. canephora (FAZUOLI, 1986; Carvalho e Fazuoli, 1993; Ferrão et al., 1999). Desenvolveram-se, também, estudos fundamentais de genética e evolução do cafeeiro (CARVALHO et al., 1985, 1991), cujos resultados são de grande importância científica e tecnológica.

Espécies vegetais perenes como o cafeeiro apresentam aspectos biológicos peculiares: ciclo reprodutivo longo; acentuada oscilação anual de produção, resultando em ciclo bienal de produção; sobreposição de gerações; expressão dos caracteres ao longo de vários anos e diferenças em precocidade e longevidade produtiva (SERA e Alves, 1999; SERA, 2001). Em termos de melhoramento genético, tais aspectos levam às seguintes conseqüências: (a) utilização do material genético selecionado para produção comer- cial por vários anos, o que demanda rigor e precisão nos métodos de seleção; (b) uso de avaliações repetidas em cada indivíduo ao longo do tempo; (c) redução na taxa de sobrevivência dos experimentos durante sua vida útil, fato que tende a gerar dados desbalanceados para uso na estimação de parâmetros genéticos e na predição dos valores genéticos aditivos e genotípicos.

Tendo em vista as conseqüências relatadas em (a), pode-se afirmar que uma ação de fundamental relevância no processo do melhoramento do cafeeiro é a predição dos valores genotípicos dos candidatos à seleção. O procedimento de predição de valores genéticos depende do conhecimento do controle genético dos caracteres sob seleção, especialmente dos parâmetros herdabilidade e repetibilidade individuais. O principal uso das estimativas de parâmetros genéticos, além do tradicional como subsídio para o planejamento de eficientes estratégias de melhoramento, está na própria predição de valores genéticos. Estimativas individuais de parâmetros genéticos, como a herdabilidade individual, são raras em café, citando-se apenas as obtidas por WALYARO e VAN DER Vossen (1979), no Quênia, e CiLAs et al. (1998), em Camarões, ambos para C. arabica, LeROY et al. (1994), na Costa do Marfim, e CILAS et al. (2000), na Costa do Marfim e Togo, ambas para C. canephora. Estimativas de parâmetros genéticos e fenotípicos, em nível de médias de linhagens são comuns no Brasil, podendo-se citar SERA $(1980,1987)$ e Sera e Alves (1999).

Em função das peculiaridades agronômicas do cafeeiro e suas conseqüências, em particular o fator (c), tem-se a necessidade do uso de métodos especiais de estimação de parâmetros genéticos e de predição de valores genéticos. Assim, os métodos tradicionais de estimação com base em análise da variância não são os mais recomendados para a análise de dados no melhoramento do cafeeiro. Atualmente, o procedimento analítico padrão recomendado para os estudos em genética quantitativa e também para a prática da seleção em plantas perenes é o REML/BLUP, ou seja, a estimação de componentes da variância por máxima verossimilhança restrita (REML) e a predição de valores genéticos pela melhor predição linear não viciada (BLUP).

Com base no exposto, o presente trabalho teve como objetivo aplicar o método REML/BLUP nos programas de melhoramento do cafeeiro, utilizando-a na 
estimação de parâmetros genéticos e predição de valores genotípicos em experimento com cultivares de C. arabica.

\section{MATERIAL E MÉTODOS}

Na aplicação do procedimento REML/BLUP considerou-se um experimento com cafeeiro instalado na Fazenda de Ensino e Pesquisa da Universidade Estadual Paulista (UNESP), Câmpus de Ilha Solteira, localizada no município de Selvíria (MS).

O experimento foi instalado em julho de 1998, com mudas provenientes de sementes de 12 cultivares de C. arabica obtidas no Instituto Agronômico, em Campinas (SP). Adotou-se o delineamento experimental em blocos ao acaso com 12 tratamentos, quatro repetições e dez plantas por parcela.

Em setembro de 2000, avaliaram-se em todas as plantas da parcela as características: altura da planta (ALT) em centímetros (cm), diâmetro do caule a $20 \mathrm{~cm}$ de altura (DIAM) em milímetros (mm) e número de pares de ramos plagiotrópicos (NRP), as quais foram analisadas. Mediu-se a altura das plantas da base até o seu ponto mais alto, o que coincide com os ramos em início de formação. O diâmetro do caule foi tomado na inserção do primeiro par de ramos plagiotrópicos, o qual foi marcado e as mensurações sempre efetuadas naquele local. O número de pares de ramos plagiotrópicos foi mensurado em toda a planta, pois o cafeeiro desenvolve-se por fluxos de pares de ramos.

As cultivares avaliadas foram: 1-IAC 4395; 2-Catuaí Amarelo IAC 62; 3-Icatu Amarelo IAC 2944; 4-Tupi IAC 1669-33; 5-Acaiá IAC 474-19; 6-Mundo Novo IAC 388-17; 7-Catuaí Vermelho IAC 144; 8-Bourbon Amarelo IAC 18; 9-Obatã IAC 1669-20; 10-Icatu Precoce IAC 3282; 11-IAC 4394; 12-Icatu Vermelho IAC 4045. Os materiais 4394 e 4395 são retrocruzamentos de Catuaí x Mundo Novo, sendo o Catuaí obtido de um cruzamento de Caturra (porte baixo) com Mundo Novo (porte alto).

Para um experimento no delineamento em blocos ao acaso com várias plantas por parcela e uma só medição por planta o seguinte modelo linear misto se aplica na avaliação de linhagens não aparentadas (conhecendo-se exatamente o parentesco entre todas as linhagens avaliadas, o procedimento de predição pode ser melhorado), trabalhando-se com dados de cada indivíduo.

\subsection{Modelo linear misto (modelo genotípico univariado)}

$$
\mathrm{y}=\mathrm{Xb}+\mathrm{Zg}+\mathrm{Wc}+\mathrm{e}
$$

em que $\mathbf{y}, \mathbf{b}, \mathbf{g}$, c e e $=$ vetores de dados individuais, dos efeitos de blocos (fixos), dos efeitos genotípicos (aleatórios), de efeitos de parcela (aleatórios) e dos erros aleatórios respectivamente; $\mathbf{X}, \mathbf{Z}$ e $\mathbf{W}=$ matrizes de incidência para $b$, g e c respectivamente.

\subsection{Distribuições e estruturas de médias e variâncias}

$$
\begin{aligned}
& \mathrm{y} \supseteq \mathrm{b}, \mathrm{V} \sim \mathrm{N}(\mathrm{Xb}, \mathrm{V}) \\
& \mathrm{g} \supseteq \sigma_{\mathrm{g}}^{2} \sim \mathrm{N}\left(0, \mathrm{I} \sigma_{\mathrm{g}}^{2}\right) \\
& \mathrm{c} \supseteq \sigma_{\mathrm{c}}^{2} \sim \mathrm{N}\left(0, \mathrm{I} \sigma_{\mathrm{c}}^{2}\right) \\
& \mathrm{e} \supseteq \sigma_{\mathrm{e}}^{2} \sim \mathrm{N}\left(0, \mathrm{I} \sigma_{\mathrm{e}}^{2}\right)
\end{aligned}
$$

ou seja:

$\operatorname{Cov}\left(\mathrm{g}, \mathrm{c}^{\prime}\right)=0 ; \operatorname{Cov}\left(\mathrm{g}, \mathrm{e}^{\prime}\right)=0 ; \operatorname{Cov}\left(\mathrm{c}, \mathrm{e}^{\prime}\right)=0$

$$
\mathrm{E}\left[\begin{array}{l}
\mathrm{y} \\
\mathrm{g} \\
\mathrm{c} \\
\mathrm{e}
\end{array}\right]=\left[\begin{array}{c}
\mathrm{xb} \\
0 \\
0 \\
0
\end{array}\right] \text { e Var }\left[\begin{array}{l}
\mathrm{y} \\
\mathrm{g} \\
\mathrm{c} \\
\mathrm{e}
\end{array}\right]=\left[\begin{array}{cccc}
\mathrm{V} & \mathrm{ZG} & \mathrm{WC} & \mathrm{R} \\
\mathrm{GZ} & \mathrm{G} & 0 & 0 \\
\mathrm{CW} & 0 & \mathrm{C} & 0 \\
\mathrm{R} & 0 & 0 & \mathrm{R}
\end{array}\right]
$$

em que:

$$
\begin{aligned}
& \mathrm{G}=\mathrm{I} \sigma_{\mathrm{g}}^{2} \\
& \mathrm{R}=\mathrm{I} \sigma_{\mathrm{c}}^{2} \\
& \mathrm{C}=\mathrm{I} \sigma_{\mathrm{e}}^{2} \\
& \mathrm{~V}=\mathrm{ZI} \sigma_{\mathrm{g}}^{2} Z^{\prime}+\mathrm{WI} \sigma_{\mathrm{c}}^{2} \mathrm{~W}^{\prime}+\mathrm{I} \sigma_{\mathrm{e}}^{2}=Z G Z^{\prime}+W C W^{\prime}+\mathrm{R}
\end{aligned}
$$

\subsection{Equações de modelo misto}

$$
\left[\begin{array}{ccc}
X^{\prime} X & X^{\prime} Z & X^{\prime} W \\
Z^{\prime} X & Z^{\prime} Z+I \lambda_{1} & Z^{\prime} W \\
W^{\prime} X & W^{\prime} Z & W^{\prime} W+I \lambda_{2}
\end{array}\right]\left[\begin{array}{l}
\hat{b} \\
\hat{g} \\
\hat{c}
\end{array}\right]=\left[\begin{array}{c}
X^{\prime} y \\
Z^{\prime} y \\
W^{\prime} y
\end{array}\right]
$$

em que:

$$
\begin{aligned}
& \lambda_{1}=\frac{\sigma_{\mathrm{e}}^{2}}{\sigma_{\mathrm{g}}^{2}}=\frac{1-\mathrm{h}_{\mathrm{a}}^{2}-\mathrm{c}^{2}}{\mathrm{~h}_{\mathrm{a}}^{2}} ; \lambda_{2}=\frac{\sigma_{\mathrm{e}}^{2}}{\sigma_{\mathrm{c}}^{2}}=\frac{1-\mathrm{h}_{\mathrm{a}}^{2}-\mathrm{c}^{2}}{\mathrm{c}^{2}} \\
& \mathrm{~h}_{\mathrm{a}}^{2}=\frac{\sigma_{\mathrm{g}}^{2}}{\sigma_{\mathrm{g}}^{2}+\sigma_{\mathrm{c}}^{2}+\sigma_{\mathrm{e}}^{2}}=\text { herdabilidade individual no sen- }
\end{aligned}
$$
tido amplo no bloco;

$$
c^{2}=\frac{\sigma_{c}^{2}}{\sigma_{g}^{2}+\sigma_{c}^{2}+\sigma_{e}^{2}}=\text { correlação devida ao ambiente }
$$

comum da parcela;

$$
\begin{aligned}
& \sigma_{\mathrm{g}}^{2}=\text { variância genotípica; } \\
& \sigma_{\mathrm{c}}^{2}=\text { variância entre parcelas; } \\
& \sigma_{\mathrm{e}}^{2}=\text { variância residual (= ambiente dentro de par- }
\end{aligned}
$$
celas). 
2.4. Estimadores iterativos dos componentes de variância por REML via algoritmo EM (Expectation-Maximization)

$\hat{\sigma}_{\mathrm{e}}^{2}=\frac{\left[\mathrm{y}^{\prime} \mathrm{y}-\hat{b}^{\prime} X^{\prime} \mathrm{y}-\hat{\mathrm{g}}^{\prime} \mathrm{Z}^{\prime} \mathrm{y}-\hat{\mathrm{c}}^{\prime} W^{\prime} \mathrm{y}\right]}{[\mathrm{N}-\mathrm{r}(\mathrm{x})]}$

$\hat{\sigma}_{\mathrm{g}}^{2}=\frac{\left[\hat{\mathrm{g}}^{\prime} \hat{\mathrm{g}}+\hat{\sigma}_{\mathrm{e}}^{2} \operatorname{tr} \mathrm{C}^{22}\right]}{\mathrm{q}}$

$\hat{\sigma}_{c}^{2}=\frac{\left[\hat{c}^{\prime} c+\hat{\sigma}_{e}^{2} \operatorname{tr} C^{33}\right]}{s}$ em que:

$\mathrm{C}^{22}$ e $\mathrm{C}^{33}$ advém de:

$C^{-1}=\left[\begin{array}{lll}C_{11} & C_{12} & C_{13} \\ C_{21} & C_{22} & C_{23} \\ C_{31} & C_{32} & C_{33}\end{array}\right]^{-1}=\left[\begin{array}{lll}C^{11} & C^{12} & C^{13} \\ C^{21} & C^{22} & C^{23} \\ C^{31} & C^{32} & C^{33}\end{array}\right]$

$C=$ matriz dos coeficientes das equações de modelo misto;

$\mathrm{tr}=$ operador traço matricial;

$\mathrm{r}(\mathrm{x})=$ posto da matriz $\mathrm{X}$;

$\mathrm{N}, \mathrm{q}, \mathrm{s}=$ número total de dados, número de linhagens e número de parcelas respectivamente.

Realizaram-se as análises com o uso do software DFREML, desenvolvido por MEYER (1998).

Em termos citogenéticos, o comportamento de indivíduos alopoliplóides, como em C. arabica, é muito similar àquele apresentado por indivíduos diplóides. Assim, em genética quantitativa, esta espécie pode ser tratada como se tratam as espécies diplóides (WRICKE e WEBER, 1986).

A decomposição do componente de variância $\sigma_{\mathrm{g}}^{2}$ pode ser realizada segundo dois modelos, ignorando a epistasia:

Modelo 1 (COCKERHAM, 1954)

$\sigma_{\mathrm{g}}^{2}=(1+\mathrm{F}) \sigma_{\mathrm{a}}^{2}+\left(1-\mathrm{F}^{2}\right) \sigma_{\mathrm{d}}^{2}$

Modelo 2 (COCKERHAM, 1983)

$\sigma_{\mathrm{g}}^{2}=(1+\mathrm{F}) \sigma_{\mathrm{a}}^{2}+\left(1-\mathrm{F}^{2}\right) \sigma_{\mathrm{d}}^{2}+4 \mathrm{FD}_{1}+\mathrm{FD}_{2}+\mathrm{F}(1-\mathrm{F}) \mathrm{H}$

em que:

$\mathrm{F}$ = coeficiente de endogamia da linhagem;

$\sigma_{a}^{2}=$ variância genética aditiva;

$\sigma_{d}^{2}=$ variância genética de dominância;

$\mathrm{D}_{1}=$ covariância entre os efeitos aditivos dos alelos e os efeitos de dominância dos homozigotos (este componente assume valores negativos para freqüências dos alelos favoráveis inferiores a 0,5 );

$\mathrm{D}_{2}=$ variância dos efeitos de dominância dos homozigotos;

$\mathrm{H}$ = soma de quadrados da depressão endogâmica de cada loco.

O modelo 2 é mais geral e válido para populações com quaisquer freqüências alélicas; já o modelo 1, para populações com freqüências alélicas intermediárias (em torno de 0,5 ). No último caso, $\mathrm{D}_{1}=\mathrm{D}_{2}=0$ e $\sigma_{d}^{2}=H$, empregando-se a expressão do modelo 2, obtém-se a expressão do modelo 1.

No presente caso, pelo método 2 , o conteúdo de $\sigma_{\mathrm{g}}^{2}$ equivale a $\sigma_{\mathrm{g}}^{2}=2 \sigma_{\mathrm{a}}^{2}+4 \mathrm{D}_{1}+\mathrm{D}_{2}$ e, pelo modelo 1 , a $\sigma_{\mathrm{g}}^{2}=2 \sigma_{\mathrm{a}}^{2}$. Assim, as definições da herdabilidade individual no sentido amplo $\left(\mathrm{h}_{\mathrm{a}}^{2}\right)$, segundo os dois modelos equivalem a:

$$
\hat{h}_{\mathrm{a}_{1}}^{2}=\frac{\hat{\sigma}_{\mathrm{g}}^{2}}{\hat{\sigma}_{\mathrm{g}}^{2}+\hat{\sigma}_{\mathrm{c}}^{2}+\hat{\sigma}_{\mathrm{e}}^{2}}=\frac{2 \hat{\sigma}_{\mathrm{a}}^{2}}{2 \hat{\sigma}_{\mathrm{a}}^{2}+\hat{\sigma}_{\mathrm{c}}^{2}+\hat{\sigma}_{\mathrm{e}}^{2}}: \text { herdabilidade indi- }
$$
vidual no sentido amplo, segundo o modelo 1;

$$
\hat{h}_{\mathrm{a}_{2}}^{2}=\frac{\hat{\sigma}_{\mathrm{g}}^{2}}{\hat{\sigma}_{\mathrm{g}}^{2}+\hat{\sigma}_{\mathrm{c}}^{2}+\hat{\sigma}_{\mathrm{e}}^{2}}=\frac{2 \hat{\sigma}_{\mathrm{a}}^{2}+4 \hat{\mathrm{D}}_{1}+\hat{\mathrm{D}}_{2}}{2 \hat{\sigma}_{\mathrm{a}}^{2}+4 \hat{\mathrm{D}}_{1}+\hat{\mathrm{D}}_{2}+\hat{\sigma}_{\mathrm{c}}^{2}+\hat{\sigma}_{\mathrm{e}}^{2}}: \text { herdabili- }
$$

dade individual no sentido amplo, segundo o modelo 2;

Assumindo o modelo (1) ou, alternativamente, tomando-se $4 \mathrm{D}_{1}+\mathrm{D}_{2}=0$, pode-se obter uma estimativa para a herdabilidade individual no sentido restrito em uma população não endógama, pela expressão:

$$
\hat{\mathrm{h}}^{2}=\frac{1}{2} \hat{\mathrm{h}}_{\mathrm{a}}^{2}
$$

A partir da $h_{a}^{2}$ - herdabilidade individual - e do componente $c^{2}$, a "herdabilidade" ao nível de médias de cultivares para cada cultivar é dada por:

$$
\hat{h}_{a_{i}}^{2}=\frac{n_{i} b_{i} h_{a}^{2}}{1+\left(n_{i}-1\right)\left(h_{a}^{2}+c^{2}\right)+\left(b_{i}-1\right) n_{i} h_{a}^{2}}: \text { "herdabilidade" }
$$
ao nível de médias para a cultivar $\mathrm{i}$;

$\mathrm{n}_{\mathrm{i}}$ e $\mathrm{b}_{\mathrm{i}}=$ número de plantas por parcela e de blocos associados à avaliação da cultivar $i$.

Com números desiguais de plantas por parcela, a expressão de $\hat{h}_{\mathrm{am}_{\mathrm{i}}}^{2}$ é dada por:

$$
\hat{h}_{a m_{i}}^{2}=\frac{\sum_{j=1}^{b_{i}} n_{i} h_{a}^{2}}{1+\left(\sum_{j=1}^{b_{i}} \frac{n_{i}}{b_{i}}-1\right)\left(h_{a}^{2}+c^{2}\right)+\left(b_{i}-1\right)\left(\sum_{j=1}^{b_{i}} \frac{n_{i}}{b_{i}}\right) h_{a}^{2}}
$$

No caso da experimentação com $n=$ uma planta por parcela, a expressão para $\mathrm{h}_{\mathrm{am}_{\mathrm{i}}}^{2}$ é dada por:

$$
h_{a_{i}}^{2 *}=\frac{b_{i} h_{a}^{2}}{1+\left(b_{i}-1\right) h_{a}^{2}}=\frac{b_{i}}{b_{i}+\frac{1-h_{a}^{2}}{h_{a}^{2}}}=\frac{b_{i}}{b_{i}-\lambda^{\prime}}
$$


em que $\lambda=\frac{1-\mathrm{h}_{\mathrm{a}}^{2}}{\mathrm{~h}_{\mathrm{a}}^{2}}$

Esta expressão foi utilizada na determinação do número adequado de indivíduos por cultivar a ser adotado na experimentação visando maximizar a acurácia seletiva.

No presente caso, a acurácia na predição dos valores genotípicos das cultivares é dada por $\hat{\mathrm{rg}}_{\mathrm{g} \mathrm{i}}=\left[\hat{\mathrm{h}}_{\mathrm{am}}^{2}\right]^{1 / 2}$, com $\hat{\mathrm{r}}_{\mathrm{gg} \mathrm{i}}$ entre 0 e 1 .

A variância do erro de predição dos valores genotípicos das cultivares é dada por $\operatorname{Var}(\hat{\mathrm{g}})=\left(1-\hat{h}_{\mathrm{am}}^{2}{ }_{\mathrm{i}}\right) \hat{\sigma}_{\mathrm{g}}^{2} \mathrm{e}$ o intervalo de confiança dos valores genotípicos preditos das cultivares, por $\hat{\mathrm{g}} \pm \mathrm{t}\left[\left(1-\hat{h}_{\mathrm{am}_{1}}^{2}\right) \hat{\sigma}_{\mathrm{g}}^{2}{ }^{1 / 2}\right.$, em que té o valor tabelado da distribuição $t$ de Student associado a determinado grau de confiança $(t=1,96$ para um intervalo construído com $95 \%$ de confiança).

\section{RESULTADOS E DISCUSSÃO}

Os resultados referentes às estimativas dos parâmetros genéticos para os três caracteres avaliados, aos 26 meses, são apresentados no quadro 1.

Verifica-se, de maneira geral, baixa variabilidade genética entre o material genético avaliado, conforme depreende-se das estimativas dos coeficientes de variação genotípica e das herdabilidades individuais
(Quadro 1). Atribui-se esse fato à idade precoce de avaliação (26 meses), considerada insuficiente para manifestar as diferenças devido ao reduzido tamanho das plantas ou, possivelmente, ao alto grau de parentesco entre as cultivares avaliadas. As estimativas dos coeficientes de herdabilidade individual no sentido amplo relatados por WALYARO e VAN DER VOSSEN (1979) obtidos da avaliação de 16 cultivares no Quênia, também apresentaram baixa magnitude (Quadro 2). É importante ressaltar que os parâmetros estimados são válidos especificamente para a população estudada, envolvendo as 12 cultivares e apenas uma avaliação aos 26 meses de campo. Outros estudos com amostragens maiores são recomendados.

Os coeficientes de herdabilidade ou de determinação genotípica individuais apresentaram baixas magnitudes, mas conduziram a coeficientes de magnitudes razoáveis ao nível de médias de cultivares, visto que os efeitos ambientais foram minimizados pelo número de repetições e de plantas por parcela. Esse fato permite uma seleção com uma precisão razoável, especialmente para os caracteres diâmetro do caule e altura da planta. Entretanto, deve-se ter em mente que esses coeficientes podem ainda estar inflacionados pelas interações genótipos $\mathrm{x}$ anos, genótipos $\mathrm{x}$ locais e genótipos $\mathrm{x}$ anos $\mathrm{x}$ locais.

Quadro 1. Estimativas de parâmetros genéticos para os caracteres altura da planta (ALT), diâmetro do caule (DIAM) e número de pares de ramos plagiotrópicos (NRP) em C. arabica, aos 26 meses

\begin{tabular}{lccc}
\hline Estimativas & ALT & DIAM & NRP \\
\hline$\hat{\mathrm{h}}_{\mathrm{a}}^{2}$ & $0,051 \pm 0,03$ & $0,118 \pm 0,04$ & $0,001 \pm 0,004$ \\
$\hat{\mathrm{h}}^{2}$ & 0,026 & 0,059 & 0,0005 \\
$\hat{\mathrm{h}}_{\mathrm{am}}^{2}(1)$ & 0,257 & 0,576 & 0,039 \\
$\hat{\mathrm{r}}_{\mathrm{g}}(1)$ & 0,507 & 0,759 & 0,197 \\
$\hat{\mathrm{c}}^{2}$ & 0,554 & 0,288 & 0,024 \\
$\hat{\sigma}_{\mathrm{g}}^{2}$ & 17,0047 & 1,8293 & 0,1270 \\
$\hat{\sigma}_{\mathrm{y}}^{2}$ & 331,1068 & 15,5122 & 103,2633 \\
$\hat{\sigma}_{\mathrm{c}}^{2}$ & 183,4041 & 4,4598 & 2,5224 \\
$\hat{\sigma}_{\mathrm{e}}^{2}$ & 130,6980 & 9,2231 & 100,6139 \\
$\mathrm{CVg}(\%)$ & 5,37 & 9,72 & 1,75 \\
Média & $76,83 \pm 7,01 \mathrm{~cm}$ & $13,92 \pm 1,16 \mathrm{~mm}$ & $20,31 \pm 1,77$ \\
\hline
\end{tabular}

${ }^{1}$ ) Estimativas considerando o estande completo: $\mathrm{n}=10$ plantas por parcela e $\mathrm{b}=4$ blocos.

$\hat{\mathrm{h}}_{\mathrm{a}}^{2}$ : herdabilidade individual no sentido amplo; $\hat{\mathrm{h}}^{2}:$ herdabilidade individual no sentido restrito; $\hat{\mathrm{h}}_{\mathrm{am}}^{2}:$ herdabilidade ao nível de média de cultivares; $\hat{r}_{g} \hat{g}:$ acurácia da predição de valores genotípicos; $\hat{c}^{2}$ : correlação devida ao ambiente comum da parcela, $\hat{\sigma}_{\mathrm{g}}^{2}:$ variância genotípica; $\hat{\sigma}_{\mathrm{y}}^{2}$ : variância fenotípica; $\hat{\sigma}_{\mathrm{c}}^{2}$ : variância entre parcelas; $\hat{\sigma}_{\mathrm{e}}^{2}$ : variância ambiental dentro de parcelas; CVg: coeficiente de variação genotípica. 
Estimativas da herdabilidade no sentido restrito ou herdabilidade dos efeitos aditivos são muito importantes no contexto da seleção recorrente, enfatizada para o melhoramento do cafeeiro por RAMALHO et al. (1999).

As estimativas de $h_{a^{\prime}}^{2}$ herdabilidade individual e $c^{2}$ são relevantes para: (a) uso no procedimento de predição de valores genotípicos das cultivares quando houver dados desbalanceados - por exemplo, sobrevivência diferenciada das cultivares; (b) uso no correto delineamento (número de repetições e de plantas por parcela) de experimentos visando à acurácia seletiva; (c) cômputo da acurácia associada à avaliação de cada cultivar; (d) cômputo do intervalo de confiança associado ao valor genotípico predito de cada cultivar.

Devido à maior variabilidade $(\mathrm{CVg})$ e coeficiente de herdabilidade da variável diâmetro do caule no presente estudo (Quadro 2), obtiveram-se os valores genotípicos (e seus intervalos de confiança) das cultivares para tal caráter (Quadro 3). O caráter diâmetro do caule tem-se mostrado correlacionado geneticamente com a produção de café e seus componentes (Walyaro e Van der Vossen, 1979; Leroy et al., 1994; Cilas et al., 1998), fato que ressalta a importância desse caráter. WALYARO e VAN DER VoSsen (1979) sugerem um índice de seleção com base no diâmetro e na produção da $1 .^{\mathrm{a}}$ e 2 . $^{\mathrm{a}}$ safras, visando ao ganho de tempo necessário para completar uma geração de seleção, de 10 para duas safras.

Verifica-se que apenas três cultivares, Catuaí Amarelo, Icatu Vermelho e Catuaí Vermelho, apresentaram valores genotípicos para diâmetro do caule superiores à média geral. Constata-se também que, quando se considera a sobreposição dos intervalos de confiança dos valores genotípicos preditos, várias cultivares mostram-se estatisticamente iguais, a 95\% de confiança (Quadro 3).

Quadro 2. Estimativas médias das herdabilidades individuais no sentido amplo $\left(\hat{\mathrm{h}}_{\mathrm{a}}^{2}\right)$ e restrito $\left(\hat{\mathrm{h}}^{2}\right)$ para vários caracteres em cafeeiros, obtidos de literatura

\begin{tabular}{|c|c|c|c|}
\hline Caráter & $\hat{\mathrm{h}}_{\mathrm{a}}^{2}$ & $\hat{h}^{2}$ & Referência \\
\hline \multicolumn{4}{|c|}{ Coffea arabica } \\
\hline Altura da planta & 0,13 & - & WALYARO e VAN DER VOSSEN (1979) \\
\hline Circunferência do caule & 0,35 & - & WALYARO e VAN DER VOSSEN (1979) \\
\hline Número de ramos primários & 0,08 & - & WALYARO e VAN DER VOSSEN (1979) \\
\hline Número de frutos por nó & 0,08 & - & WALYARO e VAN DER VOSSEN (1979) \\
\hline Inflorescências por nó & 0,10 & - & WALYARO e VAN DER VOSSEN (1979) \\
\hline Flores por inflorescências & 0,10 & - & WALYARO e VAN DER VOSSEN (1979) \\
\hline Peso de frutos/árvore* & 0,17 & - & WALYARO e VAN DER VOSSEN (1979) \\
\hline Altura da planta & 0,49 & 0,37 & CILAS et al. (1998) \\
\hline Diâmetro do caule & 0,22 & 0,01 & CILAS et al. (1998) \\
\hline Número de ramos primários & 0,16 & 0,04 & CILAS et al. (1998) \\
\hline Peso de frutos/árvore ${ }^{* *}$ & 0,40 & 0,26 & CILAS et al. (1998) \\
\hline \multicolumn{4}{|c|}{ Coffea canephora } \\
\hline Altura da planta & - & 0,37 & LEROY et al. (1994) \\
\hline Diâmetro do caule & - & 0,24 & LEROY et al. (1994) \\
\hline Número de ramos plagiotrópicos & - & 0,43 & LEROY et al. (1994) \\
\hline Peso de grãos - 1. ${ }^{a}$ safra & - & 0,28 & LEROY et al. (1994) \\
\hline Peso de grãos - 2. ${ }^{a}$ safra & - & 0,27 & LEROY et al. (1994) \\
\hline Peso de grãos - 3. ${ }^{\mathrm{a}}$ safra & - & 0,15 & LEROY et al. (1994) \\
\hline Peso de grãos - $4 .^{\mathrm{a}}$ safra & - & 0,14 & LEROY et al. (1994) \\
\hline Peso acumulado de grãos & - & 0,38 & LEROY et al. (1994) \\
\hline Elasticidade da madeira & 0,30 & - & CILAS et al. (2000) \\
\hline
\end{tabular}

*,**: Produção acumulada de duas e quatro safras respectivamente. 
Quadro 3. Valores genotípicos preditos $(\hat{\mu}+\hat{\mathrm{g}})$ e seus intervalos de confiança $(95 \%)$ para o caráter diâmetro do caule ( $\mathrm{cm})$ nas 12 cultivares de café

\begin{tabular}{cccc}
\hline Ordem & Cultivar & $\hat{\mu}+\hat{\mathrm{g}}$ & Intervalos de confiança \\
\hline 1 & 2 - Catuaí Amarelo IAC 62 & 16,8571 & $15,13-18,58$ \\
2 & 12 - Icatu Vermelho IAC 4045 & 14,8217 & $13,09-16,55$ \\
3 & 7 - Catuaí Vermelho IAC 144 & 14,0540 & $12,33-15,78$ \\
4 & 10 - Icatu Precoce IAC 3282 & 13,8797 & $12,15-15,61$ \\
5 & 1 - IAC 4395 & 13,7284 & $12,00-15,45$ \\
6 & 8- Bourbon Amarelo IAC 18 & 13,5743 & $11,85-15,30$ \\
7 & 11 - IAC 4394 & 13,5354 & $11,81-15,26$ \\
8 & 5- Acaiá IAC 474-19 & 13,4173 & $11,69-15,14$ \\
9 & 3- Icatu Amarelo IAC 2944 & 13,3323 & $11,61-15,06$ \\
10 & 9 - Obatã IAC 1669-20 & 13,3092 & $11,58-15,03$ \\
11 & 6- Mundo Novo IAC 388-17 & 13,2992 & $11,57-15,02$ \\
12 & 4- Tupi IAC 1669-33 & 13,2315 & $11,51-14,96$ \\
\hline
\end{tabular}

$\hat{\mu}=13,92$ mm: média geral.

A acurácia ou correlação entre os valores genotípicos preditos e os verdadeiros equivaleu, em média, a 0,76 (Quadro 1). Entretanto, pode-se aumentá-la por meio de uma experimentação mais adequada, mantendo-se o mesmo tamanho do experimento. No quadro 4 são apresentadas as acurácias que seriam obtidas considerando-se diferentes tamanhos de parcela e número de repetições na experimentação.

Verifica-se que, para a herdabilidade individual e $\mathrm{c}^{2}$ considerados, é preferível adotar parcelas menores e maior número de repetições ou blocos. Por exemplo, adotando-se parcelas de duas plantas e 20 repetições, a acurácia seletiva (que varia de 0 a 1 ) aumenta de 0,76 a 0,90 , em relação ao uso de quatro repetições e 10 plantas por parcela. Adicionalmente, é provável que com a redução do número de plantas por parcela, a própria herdabilidade individual no bloco $\left(\mathrm{h}_{\mathrm{a}}^{2}\right)$ aumente devido ao menor tamanho e maior homogeneidade do bloco, fato que poderá também contribuir para o aumento da acurácia seletiva. É importante relatar que, atualmente, parcelas pequenas têm sido adotadas em programas de melhoramento do cafeeiro no mundo, destacando-se os programas realizados na Costa do Marfim e em Camarões, nos quais empregam-se parcelas de uma planta (LeROY et al., 1994; CiLAS et al., 1998).

O principal enfoque deste trabalho referiu-se à aplicação de um procedimento generalizado de estimação de parâmetros genéticos e de predição de valores genéticos no melhoramento do cafeeiro. O modelo apresentado é aplicável à análise de um único experimento, desbalanceado ou não. Entretanto, vários mo- delos podem ser aplicados, sobretudo os que visam à análise conjunta de vários experimentos em presença de interação genótipo $x$ ambiente, permitindo a seleção de material genético para ambientes específicos ou para um ambiente médio (RESENDE et al., 1999); à análise com medidas repetidas em várias safras em cada indivíduo, empregando um modelo de repetibilidade (RESENDE e DiAs, 2000), dentre outros. Também, o próprio modelo utilizado pode ser empregado de maneira mais eficiente quando se conhece a genealogia completa de todo o material utilizado. Desse modo, os vários parentescos existentes são considerados matrizes de correlação genética intraclasse entre

Quadro 4. Estimativas da herdabilidade no sentido amplo ao nível de média de linhagens $\left(\mathrm{h}_{\mathrm{am}}^{2}\right)$ e da acurácia de valores genotípicos $\left(r_{g} \hat{g}\right)$ para a característica diâmetro do caule, em função da variação do número de plantas por parcela (n) e de blocos (b) empregados na experimentação, considerando $\mathrm{nb}=40$ e os parâmetros herdabilidade individual no sentido amplo $\left(\mathrm{h}_{\mathrm{a}}^{2}\right)$ igual a 0,12 e correlação devida ao ambiente comum da parcela $\left(c^{2}\right)$ igual a 0,29

\begin{tabular}{rrrr}
\hline $\mathrm{b}$ & $\mathrm{n}$ & $\mathrm{h}_{\mathrm{am}}^{2}$ & $\mathrm{r}_{\mathrm{gg}}$ \\
\hline 4 & 10 & 0,58 & 0,76 \\
5 & 8 & 0,62 & 0,79 \\
8 & 5 & 0,70 & 0,84 \\
10 & 4 & 0,73 & 0,85 \\
20 & 2 & 0,80 & 0,90 \\
40 & 1 & 0,84 & 0,92 \\
\hline
\end{tabular}


os indivíduos em avaliação, matrizes estas que são incorporadas aos estimadores e preditores.

A análise de modelos lineares mistos (REML/BLUP) pode ser aplicada também ao melhoramento do $C$. canephora, espécie alógama, visando à seleção de indivíduos para propagação por semente ou para propagação vegetativa. Nesse caso, os estimadores e preditores apresentados por RESENDE e FERNANDES (1999) são adequados. A propagação vegetativa pode ser empregada com vantagem também para o C. arabica, visto que parece existir heterose para os principais caracteres nessa espécie (Cilas et al., 1998). Assim, os indivíduos heterozigóticos com manifestação de heterose, resultantes da hibridação entre linhagens superiores, poderiam ser propagados vegetativamente na geração $F_{1}$ ou $F_{2}$, possibilitando ganhos genéticos superiores à utilização de linhas puras (RESENDE e MEDRADO, 1994).

É importante relatar as principais vantagens do uso do método de modelos mistos (REML/BLUP) na simultânea estimação de parâmetros genéticos e predição de valores genotípicos no melhoramento do cafeeiro: (a) pode ser aplicado a dados desbalanceados; (b) não exige dados obtidos sob estruturas rígidas de experimentação; (c) permite utilizar simultaneamente um grande número de informações provenientes de vários experimentos, gerando estimativas mais precisas; (d) corrige os dados para os efeitos ambientais e prediz de maneira precisa e não viciada os valores genotípicos, conduzindo à maximização do ganho genético com seleção. Em função disso, permite a utilização de conjuntos de dados que foram tomados, normalmente, dentro dos programas de melhoramento, mas que nunca foram utilizados na estimação de parâmetros genéticos, por questão de deficiência de metodologia apropriada que contemplasse, sobretudo, o desbalanceamento.

Embora haja, atualmente, um método adequado para a análise de dados desbalanceados, é importante observar que não se justifica a instalação de experimentos desbalanceados. Pelo contrário, todas as técnicas experimentais apregoadas tradicionalmente devem ser seguidas. Os dados obtidos nos programas de melhoramento e que geram informações desbalanceadas devem, porém, ser analisados conjuntamente e não, simplesmente, arquivados.

\section{CONCLUSÕES}

1. Constatou-se baixa variabilidade genética entre as cultivares para os caracteres altura da planta, diâmetro do caule e número de ramos plagiotrópicos, avaliados aos 26 meses em Selvíria (MS).
2. Apenas as cultivares Catuaí Amarelo, Icatu Vermelho e Catuaí Vermelho apresentaram valores genotípicos preditos para o diâmetro do caule superiores à média geral desse caráter.

3. A acurácia na avaliação dos valores genotípicos das cultivares para o caráter diâmetro do caule equivaleu, em média, a $76 \%$.

4. A adoção de uma experimentação com duas plantas por parcela e 20 repetições poderá elevar a $90 \%$ a acurácia seletiva para o caráter diâmetro do caule.

5. O método de modelos mistos (REML/BLUP) mostrou-se adequado à estimação de parâmetros genéticos e predição de valores genotípicos no melhoramento do cafeeiro, podendo ser empregado rotineiramente.

\section{REFERÊNCIAS BIBLIOGRÁFICAS}

CARVALHO, A.; FAZUOLI, L.C. Café. In: FURLANI, A.M.C.; VIEGAS, G.P.O. Melhoramento de plantas no Instituto Agronômico. Campinas: Instituto Agronômico, 1993. p.29-76.

CARVALHO, A.; MEDINA-FILHO, H.P.; FAZUOLI, L.C. Evolução e melhoramento do cafeeiro. In: COLÓQUIO SOBRECITOGENÉTICA EEVOLUÇÃODEPLANTAS, 10., 1984, Piracicaba. Anais... Ribeirão Preto: Sociedade Brasileira de Genética, 1985, p.215-234.

CARVALHO, A.; MEDINA-FILHO, H.P.; FAZUOLI, L.C.; GUERREIRO-FILHO, O; LIMA, M.M.A. Aspectos genéticos do cafeeiro. Revista Brasileira de Genética, Ribeirão Preto, v.14, n.1, p.135-183, 1991.

CILAS, C.; BOUHARMONT, P.; BOCCARA, M.; ESKES, A.B.; BARADAT, P. Prediction of genetic value for coffee production in Coffea arabica from a half-diallel with lines and hybrids. Euphytica, Dordrecht, v.104, p.49-59, 1998.

CILAS, C.; MONTAGNON, C.; BERTRAND, B.; GODIN, C. Wood elasticity of several Coffea canephora clones. A new trait to be included in selection schemes. Agronomie, Paris, v.20, p.439-444, 2000.

COCKERHAM, C.C. An extension of the concept of partiotioning hereditary variance for analysis of covariances among relatives when epistasis is present. Genetics, Baltimore, v.39, p.859-882, 1954.

COCKERHAM, C.C. Covariances of relatives from self fertilization. Crop Science, Madison, v.23, p.1177-1180, 1983.

FAZUOLI, L.C. Genética e melhoramento do cafeeiro. In: RenA, A.B.; Malavolta, E.; RochA, M.; YamadA, T. Cultura do cafeeiro: fatores que afetam a produtividade. Piracicaba: Associação Brasileira para Pesquisa da Potassa e do Fosfato, 1986. p.86-113. 
FERRÃO, R.G.; FONSECA, A.F.A.; FERRÃO, M.A.G. Programas de melhoramento genético de café robusta no Brasil. In: SIMPÓSIO DE ATUALIZAÇÃO EM GENÉTICA E MELHORAMENTO DE PLANTAS, 3., 1999, Lavras. Anais... Lavras: UFLA, 1999. p.50-64.

LEROY, T.; MONTAGNON, C.; CILAS, C.; CHARRIER, A.; ESKES, A.B. Reciprocal recurrent selection applied to Coffea canephora. II. Estimation of genetic parameters. Euphytica, Dordrecht, v.74, p.121-128, 1994.

MENDES, A.N.G. Métodos de melhoramento aplicados na cultura do cafeeiro. In: SIMPÓSIO DE ATUALIZAÇÃO EM GENÉTICA E MELHORAMENTO DE PLANTAS, 3., 1999, Lavras. Anais... Lavras: UFLA, 1999. p.18-35.

MEYER, K. DFREML-Version 3.0 User Notes. ArmidaleAustralia: Institute of Animal Genetics of Scotland/ Animal Genetics and Breeding Unit of the University of New England, 1998. 31p.

RAMALHO, M.A.P.; GONÇALVES, F.M.A.; SOUZASOBRINHO, F. Emprego da seleção recorrente no melhoramento do cafeeiro. In: SIMPÓSIO DE ATUALIZAÇÃO EM GENÉTICA E MELHORAMENTO DE PLANTAS, 3., 1999, Lavras. Anais... Lavras: UFLA, 1999. p.66-81.

RESENDE, M.D.V.; DIAS, L.A.S. Aplicação da metodologia de modelos mistos (REML/BLUP) na estimação de componentes de variância e predição de valores genéticos aditivos e genotípicos em espécies frutíferas. Revista Brasileira de Fruticultura, Jaboticabal, v.22, n.1, p.44-58, 2000.

RESENDE, M.D.V. de; FERNANDES, J.S.C. Procedimento BLUP individual para delineamentos experimentais aplicados ao melhoramento florestal. Revista de Matemática e Estatística, São Paulo, v.17. p.89-107, 1999.

RESENDE, M.D.V. de; FERNANDES, J.S.C.; SIMEÃO, R.M. BLUP individual multivariado em presença de interação genótipo $\mathrm{x}$ ambiente para delineamentos experimentais repetidos em vários ambientes. Revista de Matemática e Estatística, São Paulo, v.17. p.209-228, 1999.
RESENDE, M.D.V. de; MEDRADO, M.J.S. Aspectos metodológicos no melhoramento genético de Leucena leucocephala, uma espécie florestal autógama. In: CONGRESSO BRASILEIRO SOBRE SISTEMAS AGROFLORESTAIS, 1., 1994, Porto Velho. Anais... Colombo: EMBRAPA-CNPF, 1994. p.233-348. (EMBRAPA-CNPF, Documentos, 27)

SAKIYAMA, N.S; PEREIRA, A.A.; ZAMBOLIM, L. Melhoramento do café arábica. In: BORÉM, A. (Ed.). Melhoramento de espécies cultivadas. Viçosa: Imprensa Universitária, 1999. p.189-204.

SERA, T. Cofee genetic breeding at Iapar. Crop Breeding and Applied Biotechnology, Londrina, v.1, n.2, p. 179-200, 2001.

SERA, T. Desafios no melhoramento genético do cafeeiro. In: SIMPÓSIO DE ATUALIZAÇÃO EM GENÉTICA E MELHORAMENTO DE PLANTAS, 2., 1998, Lavras. Anais... Lavras: UFLA, 1998. p.105-122.

SERA, T. Estimação dos componentes da variância e do coeficiente de determinação genotípica da produção de grãos de café. Piracicaba, 1980. 62p. Dissertação (Mestrado) - Escola Superior de Agricultura "Luiz de Queiroz", Universidade de São Paulo.

SERA, T. Possibilidades de emprego de seleção nas colheitas iniciais de café (Coffea arabica, L. cv. Acaiá). Piracicaba, 1987. 147p. Tese (Doutorado) - Escola Superior de Agricultura "Luiz de Queiroz", Universidade de São Paulo.

SERA, T.; ALVES, S.J. Melhoramento genético de plantas perenes. In: DESTRO, D.; Montalván, R. (Eds.). Melhoramento Genético de Plantas. Londrina: Editora UEL, 1999. p.369-422.

WALYARO, D.J.; VAN DER VOSSEN, H.A.M. Early determination of yield potential in arabica coffee by applying index selection. Euphytica, Dordrecht, v.28, p.465-472, 1979.

WRICKE, G.; WEBER, W.E. Quantitative genetics and selection in plant breeding. Berlin: Walter de Gruyter. 1986. 406p. 